\title{
Damage Mapping with a Degrading Elastic Modulus Using Piezospectroscopic Coatings
}

\author{
Gregory Freihofer ${ }^{\mathrm{a}, *}$, Axel Schülzgen ${ }^{\mathrm{b}}$, Seetha Raghavan ${ }^{\mathrm{a}}$ \\ ${ }^{a}$ Mechanical and Aerospace Engineering Department, University of Central Florida, 4000 Central \\ Florida Blvd., Orlando, Florida, 32816, USA \\ ${ }^{b}$ CREOL, The College of Optics and Photonics, University of Central Florida, 4000 Central Florida \\ Blvd., Orlando, Florida, 32816, USA
}

\begin{abstract}
The development of piezospectroscopic (PS) composites has enabled the creation of a non-destructive evaluation (NDE) technique which integrates piezospectroscopy, digital image correlation (DIC) and analytical multi-scale mechanics to map the elastic modulus of a coated material. The measured elastic modulus was represented as a normal distribution with a mean value $(32.2 \mathrm{GPa})$ which is within $8 \%$ of the conventionally recorded modulus (35 GPa). Damage mechanics are applied to map elastic degradation in-situ mechanical loading with an average uncertainty $(\sim 10 G P a)$ that was sufficient in observing subsurface, progressive damage patterns which are unique to the coated material.

Keywords: Piezospectroscopy, Mechanical Properties, Multiscale Mechanics, Damage Mechanics, Non-destructive Evaluation
\end{abstract}

\section{Introduction}

Detecting damage with NDE is an important field for the diagnosis of structural health with applications ranging from aerospace [1] to civil [2] structures. Conventional NDE techniques are based on the identification of the size and location of defects. Techniques such as ultrasound [3], thermography [4], shearography [5], and others [6] are technologies that each have their own set of advantages in this regard.

\footnotetext{
${ }^{*}$ Corresponding author

Email address: Freihofer@knights.ucf.edu (Gregory Freihofer)
} 
An alternative way of monitoring damage is by quantifying the reduced mechanical properties. The presence of micro-voids or cracks create a softening effect which decreases the elastic modulus of the continuum [7]. When compared to a variety of damage measures, monitoring the degrading elastic modulus is a promising technique [8]. The degraded elastic modulus can be captured experimentally with the slope of an unloading curve or predicted with a damage model. The major weakness is that it lacks the capacity to detect local damage because a measure of the elastic modulus is conventionally a nominal or macroscopic measure.

In laboratory settings, determining nominal mechanical properties are straightforward with a load cell, strain gage, and standard test specimens. However, evaluating local mechanical properties of a structure would require a combination of stress and strain mapping. Strain mapping is available with a few techniques including electronic speckle pattern interferometry [9], Moiré interferometry [10], and digital image correlation (DIC) [11]. Stress mapping with the same flexibilities as the previously mentioned strain mapping techniques has not been available, until now, with piezospectroscopy. This work shows that piezospectroscopy, recorded over a field of view, can couple with strain maps to locally distinguish elastic moduli, thereby removing the weakness this damage measure is nominal.

Other approaches to estimate local mechanical properties are available such as atomic force microscopy $[12,13]$ and ultrasonic [14] methods. These techniques rely on other physical principles to determine the elastic modulus rather than conventional stress/strain relationships and are currently active fields of research [15, 3]. Among these, however, piezospectroscopy stands out as the one technique suitable enough for integration with full-scale mechanical load frames for standard coupon testing $[16,17]$.

Piezospectroscopy with $\mathrm{Cr}$ doped $\mathrm{Al}_{2} \mathrm{O}_{3}$ has been most widely used as a pressure sensor in diamond anvil cells [18], and as an NDE technique for monitoring the health of thermal barrier coatings [19]. The applications of piezospectroscopy has recently been expanded by the fabrication of alumina-epoxy nanocomposites with tailorable mechanical and PS properties [20]. This produced a material which can be applied as 
a compliant coating to structures for stress sensing $[21,22,23]$. In the process of holistically understanding the multi-scale mechanics of these new materials, the solution of an effective elastic modulus of the nanocomposite with the experimental PS response was discovered [24]. In this work, multi-scale mechanics are extended to a nanocomposite coating, to solve for an effective elastic modulus of the composite substrate in-situ mechanical loading.

\section{Experiment}

The PS nanocomposite coating investigated in this work was manufactured by Elantas PDG, Inc. by mixing $150 \mathrm{~nm}, \mathrm{Cr}$ doped $\alpha-\mathrm{Al}_{2} \mathrm{O}_{3}$ nanoparticles (Inframat Corp.) with $99.8 \%$ purity in epoxy to achieve a $20 \%$ volume fraction of particles. The coating was applied to a composite substrate consisting of laminated IM7-8552 unidirectional tape (57.7\% fiber volume) manufactured with a $[45 /-45 / 0 / 45 /-45 / 90 / 45 /-45 / 45 /-45]_{s}$ layup resulting in transversely isotropic properties $\left(E_{1}=E_{2}=35 \mathrm{GPa}\right)$ [25]. The coating has a very small thickness $(300 \mu \mathrm{m})$ and low modulus $(<1 \mathrm{GPa})$ which ensures it does not mechanically reinforce the composite substrate. The coated sample was machined and tested in accordance with composite open hole tension (OHT) ASTM standards [26]. The length, width, thickness and hole size of the coupon were 12, 1.5, 0.15 , and 0.25 in respectively.

The sample was loaded at a rate of $0.05 \mathrm{in} / \mathrm{min}(0.127 \mathrm{~cm} / \mathrm{min})$ and held using displacement control using a hydraulic load frame at 10 load points throughout the experiment as marked in Figure 1. This avoided creep during the higher loads, but prompted stress relaxation which was acceptable since the goal was to observe damage progression. During each hold, PS and DIC measurements were collected on the front and back side of the substrate, respectively. Both measurements can be directly compared since the substrate has a symmetric layup, shown in Figure 1.

PS data was collected with a prototype portable spectrometer system designed to be easily transported with a quick setup time [17]. Excitation was achieved using a low power, $1.5 \mathrm{~mW}$ laser of $532 \mathrm{~nm}$ wavelength in a back scattering configuration with a 


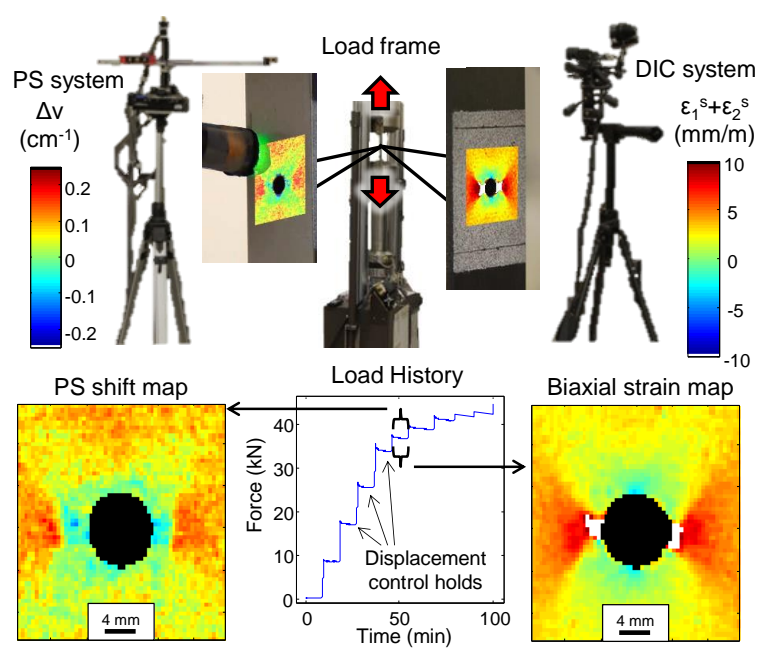

Figure 1: Both PS and DIC tripod systems are measuring the stress and strain field respectively on opposing sides of the composite coupon in a mechanical load frame. The steps within the load history graph are displacement control holds, which were necessary to prevent creep in the sample at the high failure loads during mapping the PS response. This resulted in a softening of the material during and is observed as a reduction in force during the load steps.

long working distance objective $(28 \mathrm{~mm})$. The photoluminescence $(\mathrm{PL})$ spectra were collected on a 60x60 grid during a snake scan, implemented using a synchronized translation stage, with the accumulation time for spectra at $100 \mathrm{~ms}$. The total measurement area was $25.4 \mathrm{~mm}$ squared, corresponding to a spatial resolution of $0.4 \mathrm{~mm}$ in both directions of the measurement plane. With these settings each map is collected within 8 minutes.

The DIC measurements were collected at the beginning of every hold and were relatively instantaneous. The speckle pattern, which was spray painted, had an average dot size of roughly $0.2 \mathrm{~mm}$. The DIC images were post processed to have a spatial resolution equal to that of the PS maps $(0.4 \mathrm{~mm})$. Furthermore, a post-processing algorithm was created to spatially match up the DIC and PS mapping data. In brevity, the algorithm interpolated data on a new coordinate system using the open hole as a reference. The accuracy of the pixel match up is worst in regions closest to the open hole $(\leq 0.4 \mathrm{~mm})$ but is negligible at a few pixels distance away (1-2) [27]. 


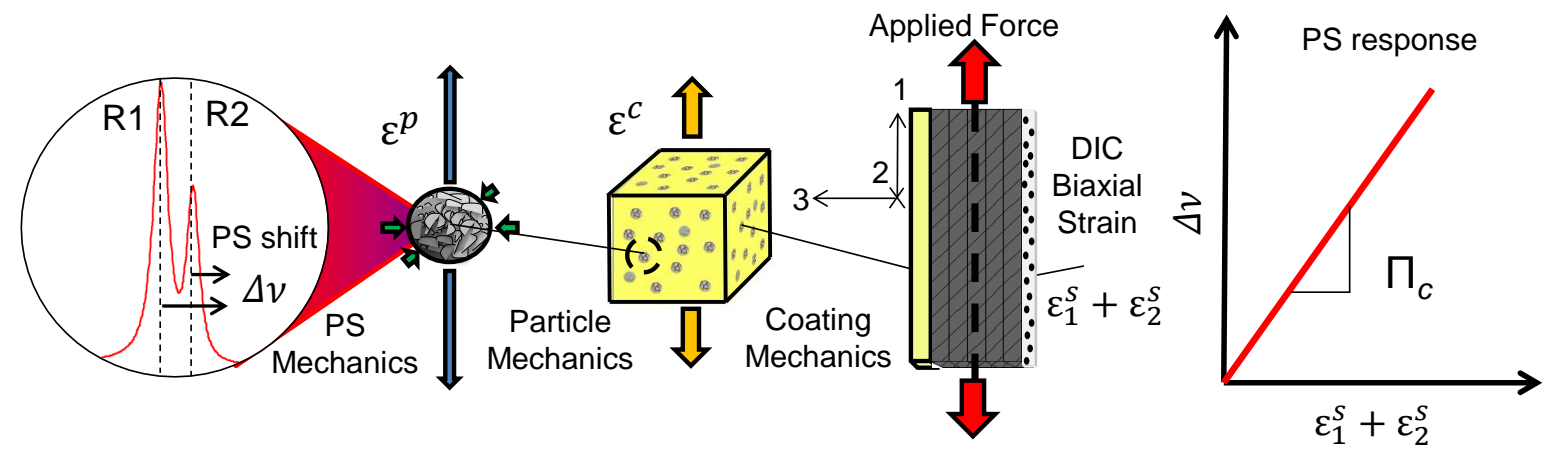

Figure 2: A summary of the multiscale mechanics to describe the nanocomposite coating problem.

\section{Multiscale Mechanics to Model the PS response}

Spatial measurements of substrate strain and PS shift were obtained simultaneously for the first time in an novel experiment [23]. The PS relationships originate from the distortion of the Ligand field around the $\mathrm{Cr}^{3+}$ substitutional impurity within the $\mathrm{Al}_{2} \mathrm{O}_{3}$ lattice. As the lattice is deformed under mechanical load, the energy levels of the Ligand field change, resulting in a frequency shift of the photo-stimulated luminescence [28]. Usually, the PS relationships are dependent on crystallographic orientation, but for a polycrystalline a material whose grain size is an order of magnitude smaller than the probed volume (i.e. laser dot size) an averaging effect takes place and simplifies the PS relationship to be a function of the first stress or strain invariant [29]. In this study, the PS shift is considered as the mean shift of the R1 and R2 spectral lines, which has been associated to the first stress invariant [24, 30].

Here, the PS response is taken as the substrate's DIC biaxial strain $\left(\epsilon_{1}^{s}+\epsilon_{2}^{s}\right)$ vs. PS shift $(\Delta \nu)$. When evaluating this relationship, a variety of mechanics have to be considered as illustrated in Figure 2. For linear elastic behavior, a PS response is described by the composite's PS coefficient $\left(\Pi_{c}\right)$ in $\Delta \nu=\Pi_{c}\left(\epsilon_{1}^{s}+\epsilon_{2}^{s}\right)$

This PS coefficient is experimentally measured for every pixel when the PS shift and DIC biaxial strain maps are combined. This experimental PS coefficient represents a combination of several mechanics, that describe the process when load is transfered from the substrate to the coating to the particle. The series of ratios that describe this 
PS coefficient are shown in Eq. (1).

$$
\Pi_{c}=\frac{\Delta \nu}{\epsilon_{i i}^{p}} \frac{\epsilon_{i i}^{p}}{\epsilon_{i i}^{c}} \frac{\epsilon_{i i}^{c}}{\epsilon_{1}^{s}+\epsilon_{2}^{s}}
$$

The first ratio encompasses the relationship between the mean R-line shift with the particle's first strain invariant $\left(\epsilon_{i i}^{p}\right)$ as shown in Eq. (2). This is a variation of the equation that commonly uses the first stress invariant $\left(\sigma_{i i}\right)$ with the PS shift by the trace of PS tensor $\left(\Pi_{i i}=7.6 \mathrm{~cm}^{-1} / G P a\right)[31]$. The first stress and strain invariant is interchangeable with the bulk modulus of the particle $\left(K^{p}\right)$ using the relation $3 K \epsilon_{i i}=$ $\sigma_{i i}$.

$$
\frac{\Delta \nu}{\epsilon_{i i}^{p}}=\Pi_{i i} K^{p}
$$

The second ratio describes the relationship between the first strain invariant of the particle and coating. This equation was derived using Eshelby's inclusion mechanics [24] which assumed a spherical particle, and both phases are isotropic. The ratio is a function of the properties for the coating $\left(E^{c}, \nu^{c}\right)$ and particle $\left(E^{p}, \nu^{p}\right)$ shown in Eq. (3).

$$
\frac{\epsilon_{i i}^{p}}{\epsilon_{i i}^{c}}=\frac{-3 E^{c}\left(2 \nu^{p}-1\right)\left(\nu^{c}-1\right)}{\left(2 \nu^{c}-1\right)\left(2 E^{c}+E^{p}-4 E^{c} \nu^{p}+E^{p} \nu^{c}\right)}
$$

The last ratio describes the relationship between the first invariant of the coating with the biaxial strain of the substrate. A number of steps are associated with obtaining this ratio which are outlined elsewhere [27] and are valid for a thin compliant coating. Briefly, the expression is obtained by equating interface strains $\left(\epsilon_{1}^{s}=\epsilon_{1}^{c}, \epsilon_{2}^{s}=\epsilon_{2}^{c}\right)$ between the isotropic coating and transversely isotropic substrate under plane stress $\left(\sigma_{3}^{s}=\sigma_{3}^{c}=0\right)$. In addition, the expression $\epsilon_{i i}^{s} /\left(\epsilon_{1}^{s}+\epsilon_{2}^{s}\right)=\left(1-2 \nu^{s}\right) /\left(1-\nu^{s}\right)$ is used which is valid for a transversely isotropic material in plane stress. The result is only a function of the Poisson's ratio for the coating $\left(\nu^{c}\right)$ and substrate $\left(\nu^{s}\right)$, given in Eq. (4).

$$
\frac{\epsilon_{i i}^{c}}{\epsilon_{1}^{s}+\epsilon_{2}^{s}}=\frac{\left(1-\nu^{s}-\nu^{s 2}\right)\left(1-\nu^{s}\right)\left(1+\nu^{s}\right)\left(1-2 \nu^{s}\right)}{\left(1-\nu^{c}-\nu^{c 2}\right)\left(1-\nu^{c}\right)^{2}\left(1+\nu^{c}\right)}
$$


The series of ratios that describe the PS coefficient have now been described and is a function as follows: $\Pi_{c}=f\left(\Pi_{i i}, E^{p}, \nu^{p}, E^{c}, \nu^{c}, \nu^{s}\right)$. After all of the applied mechanics, the PS coefficient was not a function of the elastic modulus of the substrate $\left(E^{s}\right)$. To obtain an expression which includes $E^{s}$, and solely a function thereof requires a set of assumptions.

1. The strain which the substrate experiences is directly transfered into the particles. This is appropriate with a compliant coating with low elastic modulus and thickness, providing no mechanical reinforcement. This enables the removal of the coating mechanics term and the replacement of the coating's isotropic properties with the substrate's in Eq. (3). Therefore:

$\Pi_{c}=f\left(\Pi_{i i}, E^{p}, \nu^{p}, E^{s}, \nu^{s}\right)$

2. The particle's properties are known $\left(E^{p}=400 G P a, \nu^{p}=0.23\right)$. A variation of particle properties amongst literature [32, 33, 34, 35] has resulted in a conservative uncertainty reported in the methods. $\Pi_{i i}$ is also considered a property of the particle. Therefore:

$\Pi_{c}=f\left(E^{s}, \nu^{s}\right)$

3. Isotropic damage assumes that the Poisson's ratio does not change [36], and the substrate's pristine Poisson's ratio can be estimated using classical laminate plate theory [37] $\left(\nu^{s}=0.57\right)$. Therefore:

$\Pi_{c}=f\left(E^{s}\right)$

The first assumption makes the substrate isotropic. The challenge is to modify the Eshelby tensor for a transversely isotropic media [38]. For this application, it is desired to solve the Eshelby tensor symbolically, in order to rearrange Eshelby's equation for the matrix's elastic modulus, as was done recently for an isotropic media [24]. The complexity of the explicit solution for a transversely isotropic Eshelby tensor, and lack thereof for a generic anisotropic case [39], results in a challenging undertaking to remove the isotropic substrate assumption.

With the above assumptions, a refined series of ratios can now be combined and 

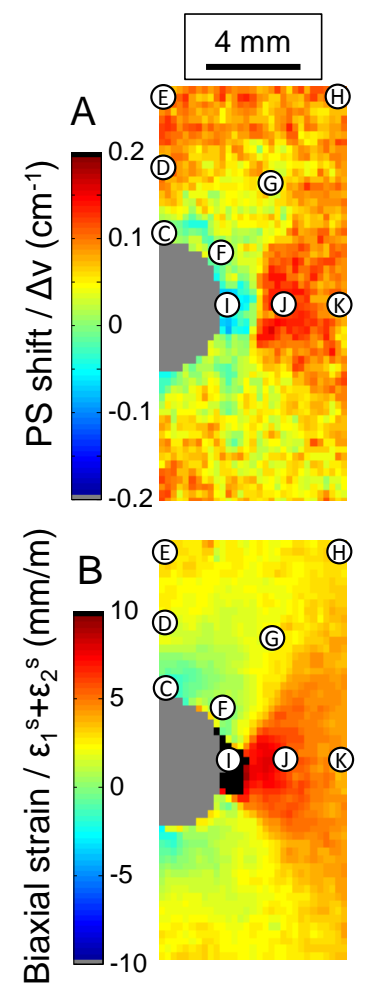
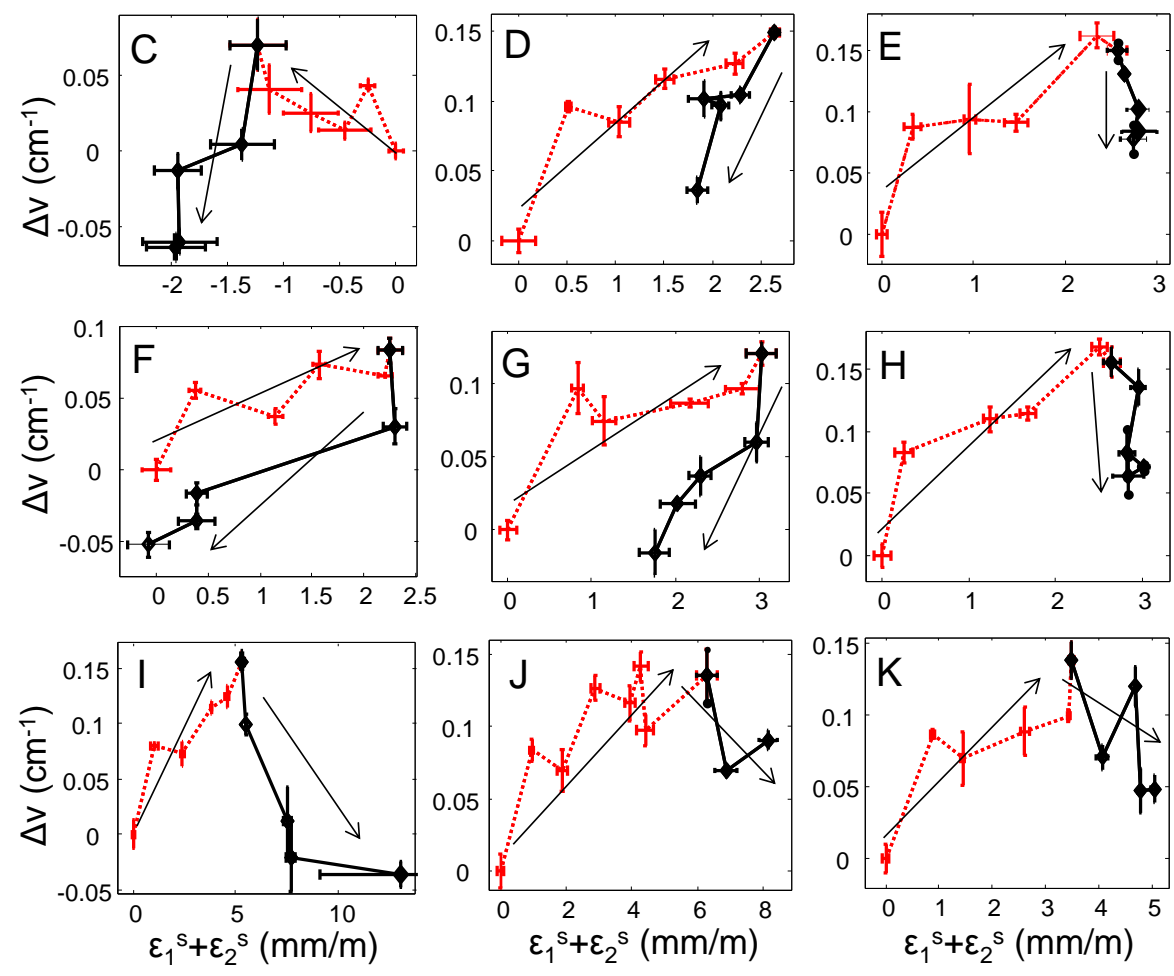

Figure 3: (A) PS shift and (B) biaxial strain maps for half of the open hole. (C to L), A variety of local PS responses, each representing a $400 \mu \mathrm{m}$ square area from indicated locations labeled on the PS and DIC maps. The $\mathrm{x}$ and y error bars represent spatial variations during an alignment and interpolation algorithm which combined the PS and DIC data sets discussed in the Appendix.

rearranged to solve for the substrate's elastic modulus. This leaves a direct solution of the substrates elastic modulus with an experimental measure of the first order PS coefficient $\left(\Pi_{c}\right)$ from the PS response.

$$
E^{s}=\frac{E^{p} \Pi_{c}\left(\nu^{s}+1\right)}{2 \Pi_{c}-3 K^{p} \Pi_{i i}\left(2 \nu^{p}-1\right)}
$$

The character of the PS responses vary depending on their location with respect to the open hole. In Figure 3, the variation in the PS responses are attributed to the non proportional and non uniform loading across the sample's surface. This, combined with the relatively few data points at low loads, makes for a challenge in determining the first order approximation of $\Pi_{c}$. 
The composite substrate will have linear elastic behavior until fracture. This is dissimilar to the high volume fraction nanocomposite coating (20\%) which has inelastic properties $[40,32]$. This explains the non-linearity in the PS response, but makes it difficult to quantify $\Pi_{c}$ for the elastic modulus calculation.

To have a robust method of obtaining $\Pi_{c}$ for every PS response, a yield function is fit to a segment of the PS response from zero load until the maximum PS shift. A Ramberg-Osgood yield function was used because it works well without a well defined yield point [41]. This yield function is normally used for a strain-stress plot, but was modified here for a strain-PS shift plot in Eq. (6). This reformulation of the RambergOsgood formula is appropriate because the PS shift is directly proportional to the strain as derived in the previous section. For brevity, $\epsilon_{b}=\epsilon_{1}+\epsilon_{2}$.

$$
\Delta \nu=\Pi_{c} \epsilon_{b}+\alpha \epsilon_{b y} \Pi_{c}\left(\frac{\epsilon_{b}}{\epsilon_{b y}}\right)^{m}
$$

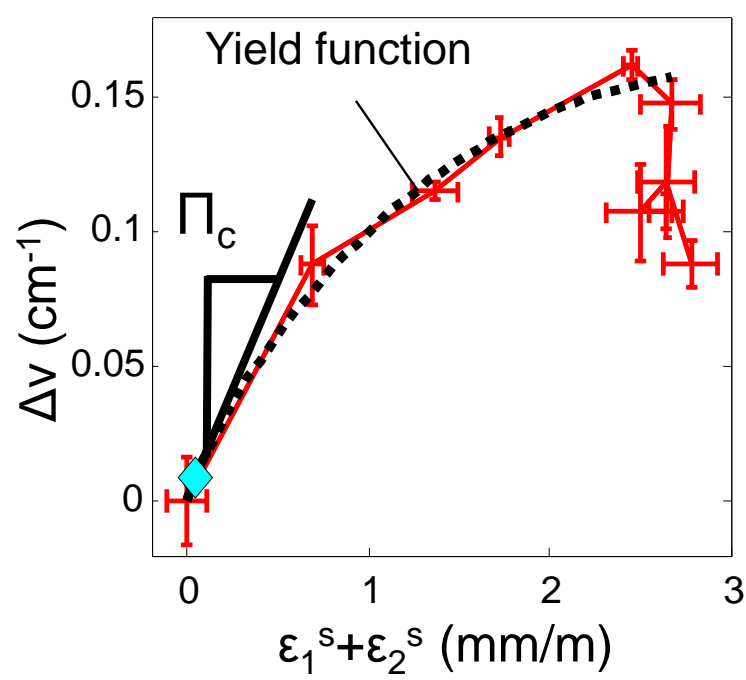

Figure 4: The yield function is marked on the PS response with a marker designating the strain at $0.025 \mathrm{~mm} / \mathrm{m}$ which the derivative was evaluated to calculate $\Pi_{c}$.

Eq. (6) was fitted to every local PS response such as shown in Figure 4. The $\Pi_{c}$ value as solved by the fitting algorithm varied significantly across the surface due to 
the sparse number of data points at low loads and was not suitable to solve for $E^{s}$. Due to the need to have a more consistent estimation of $\Pi_{c}$, The yield function was used to interpolate a derivative of the PS response at a very small strain between all of the PS responses. This would be the approximation of the first order PS coefficient before the non-linearity initiated. The strain chosen to evaluate the derivative of the yield function and compute $\Pi_{c}$ was chosen to be $0.025 \mathrm{~mm} / \mathrm{m}$ for all PS responses and is marked in Figure 4.

\section{Elastic Modulus and Damage Mapping}

The appropriate mechanics have been derived to calculate the substrate's elastic moduli $\left(E^{s}\right)$ from the composite's first order PS coefficients $\left(\Pi_{c}\right)$ and a yield function has been used to obtain a consistent measure of $\Pi_{c}$ for every PS response. Now, by applying Eq. (5), an elastic modulus map was created in Figure 5A and a corresponding histogram in Figure 5C. Due to a broad distribution within the histogram, uncertainty of the elastic modulus map was investigated before a comparison to a-priori substrate elastic moduli values.

A significant number of points on the surface were identified to have large errors from experimental sources and a systematic uncertainty, described in the Appendix. When the local uncertainty of elastic modulus calculation exceeded $15.75 \mathrm{GPa}$, or $45 \%$ of the a-priori known unnotched nominal modulus, the data points were filtered and these points on the elastic modulus map were replaced with white pixels in Figure 5B post-filter. A significant amount of data points above and below the open hole contain white pixels because the longitudinal $\left(\epsilon_{1}\right)$ and transverse $\left(\epsilon_{2}\right)$ strains are nearly equal and opposite. This also combined with uncertainty from the algorithm used to align and interpolate the data sets based as discussed in the Appendix.

In addition, small regions directly above and below the hole contained negative values of $\Pi_{c}$, resulting in a negative $E^{s}$ and they can be distinguished in Figure $5 \mathrm{~A}$ as white pixels pre-filter. These regions are the result of a compressive biaxial strain, but a tensile PS shift. Complex loading conditions associated with the non-proportional 

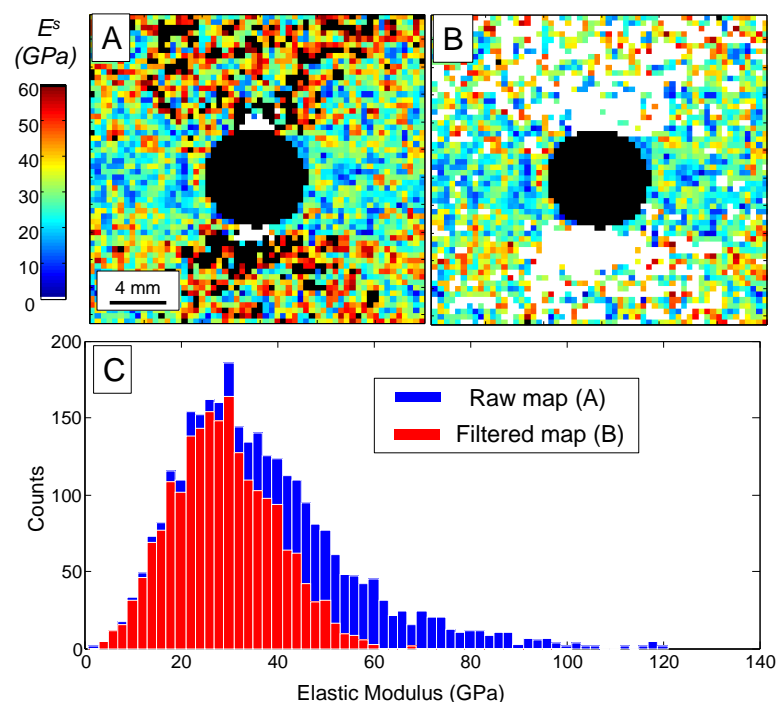

Figure 5: Elastic modulus maps $(\mathbf{A})$ before and $(\mathbf{B})$ after the experimental uncertainty filter is applied. C, The corresponding histograms of the elastic modulus across the sample's surface.

loading around the open hole are correlated to the observed negative moduli. One of the PS responses in this region that can be observed in Figure 3C.

With the uncertainty issues addressed, the PS recorded moduli were compared to a previous test of control samples (16 unnotched) which determined that the nominal elastic modulus was $35 \pm 3 G P a$ [25]. The modulus map in Figure 5A shows a broad distribution of elastic modulus values with a mean centered at $32.2 \mathrm{GPa}$ and a standard deviation of $9.4 \mathrm{GPa}$ post-filter. The large number of data points taken across the surface produced a mean that closely resembles expected values for the unnotched, underlying substrate. The large standard deviation of the PS calculated moduli are proposed to be caused by a inhomogeneous dispersion of the nanoparticles, which is still an active field of research in the material's development [42]. These agglomerations lead to local variations in particle volume fraction, and thus particle-matrix load transfer mechanics [24].

As a damage measure, the elastic degradation of a material through mechanical loading [43] comes from the reduced effective stress carrying area by the presence of a crack or defect [44]. The latter results in an increase in stress on the remaining part of 
the material and is associated with a nominal reduction in stiffness [7]. For instance, the nominal modulus of the open hole tension composite coupon was $25 G P a$, but the unnotched coupon was $35 G P a$.

Conventionally, unloading curves during a mechanical test are used to monitor a reduced elastic modulus from the presence of damage [43]. This reduction in stiffness is referred to as elastic degradation. If unloading curves were not integrated in the experimental procedure, then the unloading curves may be simulated to estimate the reduced mechanical properties. Methods of simulating these unloading curves vary in complexity, ranging from simple elastic degradation models [7] to the involved continuum damage mechanics with coupled plasticity [7]. A simple and convenient method to simulate an unloading curve is to assume no plastic deformation so all unloading curves return to the origin, or contrarily, no elastic degradation and purely plastic deformation [7].

In reality, unloading is usually associated with a coupling between the elastic degradation and plastic deformation. Here, a method for simulating unloading curves was used which included both plastic deformation and elastic degradation as shown in Figure $6 \mathrm{~A}$. The simulated unloading curves only consider plastic deformation and no elastic degradation until the max PS shift has been reached. Further loading past the max PS shift ceases plastic deformation, and initiates elastic degradation.

The slopes of the simulated unloading curves on the PS response can be readily converted into an elastic modulus value with an isotropic damage assumption [36], that is the Poisson's ratio remains constant. The degraded elastic modulus values $(\tilde{E})$ are normalized with respected to local pristine modulus $(E)$, to create a damage measure $\left(D=1-\frac{\tilde{E}}{E}\right)[8,43]$. These damage maps reveal intrinsic damage features of the composite substrate. Major turning points in the progression of damage are plotted in Figure 6 .

The first significant occurrence of damage is at $76 \%$ failure load just adjacent to the open hole. It is known a-priori that this region should be experiencing large tensile strains, and is a likely location for damage to initiate. In other work by Camanho [45], 


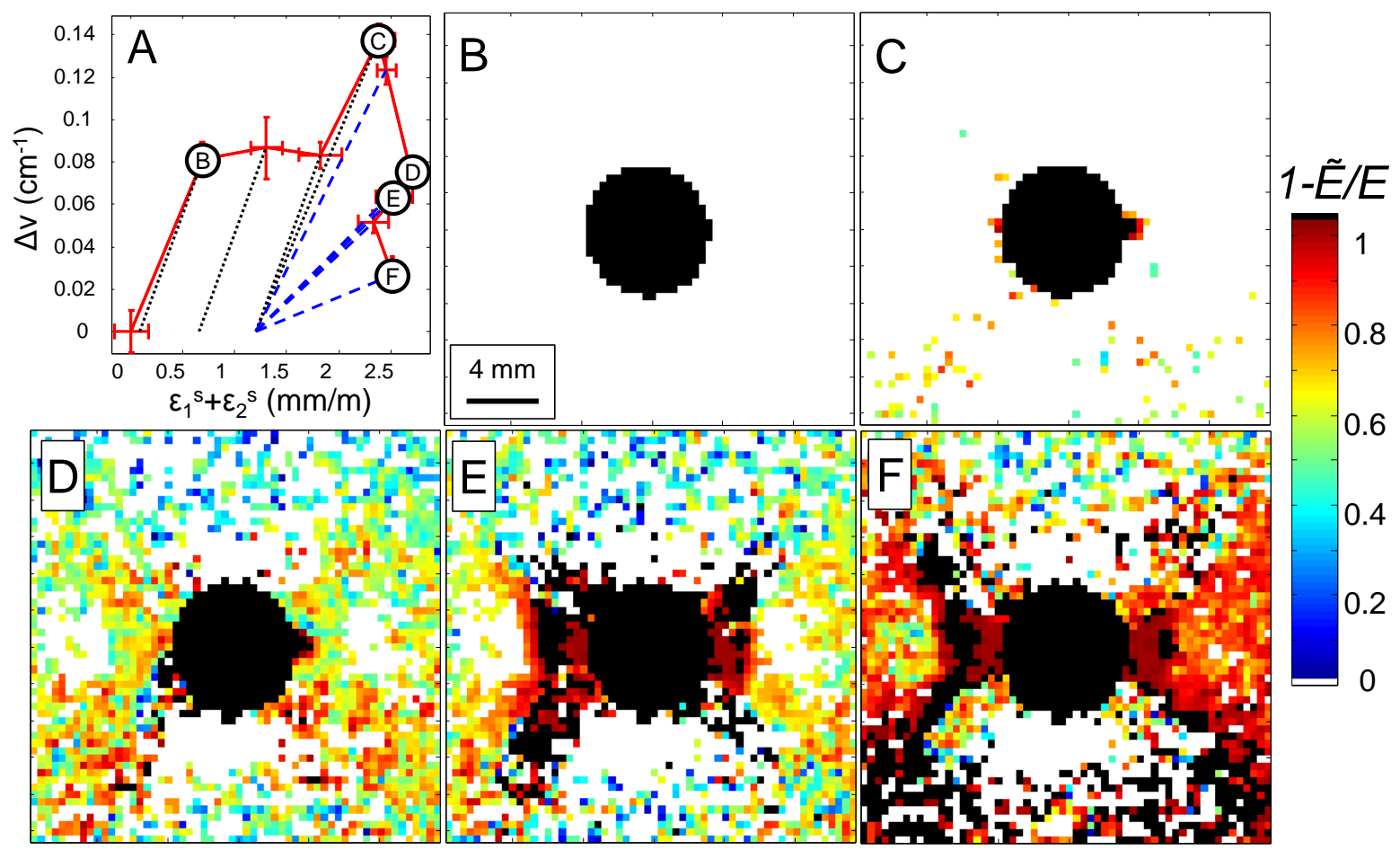

Figure 6: (A), The simulation of the unloading curves for a PS response using plastic deformation until the max PS shift, after which purely elastic degradation is initiated. Loads which exhibited significant progression of damage are shown including (B) 0, (C) 76, (D) 88, (E) 92, and (F) $96 \%$ failure load. 
a simulation using continuum damage mechanics of a transversely-isotropic open hole tension composite coupon predicted initial fiber failure in the $0^{\circ}$ ply in this exact region. Additionally, at $76 \%$ failure load, audible cracking in the sample suggest that significant damage had initiated.

The next significant progression of damage was at $88 \%$ failure load where homogeneous damage appeared across the sample's surface. This experimentally observed phenomena could be due to transverse cracking of the $90^{\circ}$ plies which occurs homogeneously through the length of the sample [46]. In addition, literature has described a redistribution of stress occurring once subsurface damage has become significant [47]. This loading point is an important turning point in the mechanical test that may indicate the onset of critical damage.

Next, at $92 \%$ failure load, a large propagation of damage occurs on both sides of the open hole. A study using X-ray inspection has revealed similar cracking patterns [47] adjacent to the open hole in a tension test for a similar composite coupon. As the higher failure loads are reached, the damage pattern resembles intra-laminate cracking of the $\pm 45^{\circ}$ plies, and is very similar to predicted crack growth patterns with numerical models [47].

\section{Conclusion}

This work has successfully applied multi-scale mechanics to a PS coating experiment to obtain, for the first time, high spatial resolution damage maps using a degrading elastic modulus. Simulation of unloading curves in-situ mechanical loading are used to estimate elastic degradation patterns which resemble intrinsic, subsurface damage patterns of the composite substrate $[47,46,45]$. In addition to locating the size and

location of damage, the ability to quantify the degradation of the elastic modulus enables the distinction of different types of damage. For example, matrix microcracking should change the equivalent modulus by a small amount in comparison to fiber failure or intra-laminate cracking. In the current study, the average uncertainty in the elastic modulus calculation was nearly $10 \mathrm{GPa}$ and results in the detection of only critical 
damage. Future experiments will be designed to lower this uncertainty to detect more subtle changes in the effective elastic properties and adapt the coating to a wide range of substrates materials. An NDE technique that provides such a comprehensive damage characterization would be very desirable.

\section{Acknowledgments}

This material is based upon work supported by the National Science Foundation under Grant No. CMMI 1130837 and the University of Central Florida Office of Research and Commercialization Inhouse Grant FY 2012. Hong Tat, Gerardo Peña, and Jordan Handler from Boeing Research \& Technology are acknowledged for technical discussions.

\section{Appendix A. Elastic Modulus Uncertainty Estimation}

A filtering process was used to remove data points with high experimental error as shown in Experimental uncertainties in the data collection varied from point to point based on a calculation of a slope $\left(\Pi_{c}\right)$, from local biaxial strain $\left(\epsilon_{b}=\epsilon_{1}+\epsilon_{2}\right)$ and PS shift data $(\Delta \nu)$ in $\Delta \nu=\Pi_{c} \epsilon_{b}^{s}$. Here, $U_{\Delta \nu}$ and $U_{\epsilon_{b}}$ were taken as the error bars of the PS response at $20 \%$ failure load, which represents the standard deviation of local spatial variations calculated during the interpolation procedure of aligning the two data sets into a global coordinate system [27]. For the PS coefficient $\left(U_{\Pi_{c}}\right)$, the systematic uncertainty various across the surface of the sample with an average value of $38.1 \mathrm{~cm}^{-1} \mathrm{~m} / \mathrm{m}$ of all the post-filtered pixels using Eq. (A.1).

$$
\begin{gathered}
U_{\Pi_{c}}^{2}=\left(\frac{\partial \Pi_{c}}{\partial \epsilon_{b}} U_{\epsilon_{b}}\right)^{2}+\left(\frac{\partial \Pi_{c}}{\partial \Delta \nu} U_{\Delta \nu}\right)^{2} \\
U_{E^{s}}^{2}=\left(\frac{\partial E^{s}}{\partial \Pi_{c}} U_{\Pi_{c}}\right)^{2}+\left(\frac{\partial E^{s}}{\partial \prod_{i i}} U_{\Pi_{i i}}\right)^{2}+\left(\frac{\partial E^{s}}{\partial E^{p}} U_{E^{p}}\right)^{2}+\left(\frac{\partial E^{s}}{\partial \nu^{p}} U_{\nu^{p}}\right)^{2}+\left(\frac{\partial E^{s}}{\partial \nu^{s}} U_{\nu^{s}}\right)^{2}
\end{gathered}
$$

This experimental uncertainty for $\Pi_{c}$ is then plugged into the systematic uncertainty for the elastic modulus calculation given in Eq. (A.2). Every independent variable has 
its own uncertainty and contributes to the total uncertainty of the elastic modulus. The uncertainties for these values and their contribution to the total uncertainty are as follows: $U_{\Pi_{i i}}=0.05 \mathrm{~cm}^{-1} / G P a, U_{E^{p}}=50 \mathrm{GPa}, U_{\nu^{p}}=0.05, U_{\nu^{s}}=0.05$, each contributing to the elastic moduli uncertainty calculation of $0.2,3.3,4.9$, and $0.8 \mathrm{GPa}$ respectively. Every pixel in the elastic modulus map has its own independent $U_{\Pi_{c}}$ value, and the mean contribution to the elastic moduli uncertainty was $8.6 \mathrm{GPa}$. With all of the independent uncertainties combined, the mean experimental uncertainty for the substrate's elastic moduli calculation $U_{E^{s}}$ was $10.5 \mathrm{GPa}$ post-filtering. With this being the major contributing factor to the uncertainty, future work aims to design specialized equipment to capture the R-line peak shifts.

[1] B. Kamsu-Foguem, Knowledge-based support in non-destructive testing for health monitoring of aircraft structures, Advanced Engineering Informatics 26 (2012) 859869.

[2] D. McCann, M. Forde, Review of NDT methods in the assessment of concrete masonry structures, NDT \& E International 34 (2001) 71-84.

[3] B. W. Drinkwater, P. D. Wilcox, Ultrasonic arrays for non-destructive evaluation: A review, NDT \& E International 39 (2006) 525-541.

[4] S. Bagavathiappan, B. Lahiri, T. Saravanan, J. Philip, T. Jayakumar, Infrared thermography for condition monitoring - a review, Infrared Physics \& Technology 60 (2013) 35-55.

[5] D. Francis, R. P. Tatam, R. M. Groves, Shearography technology and applications: a review, Measurement Science and Technology 21 (2010) 102001.

[6] Y.-K. Zhu, G.-Y. Tian, R.-S. Lu, H. Zhang, A review of optical NDT technologies, Sensors 11 (2011) 7773-7798.

[7] I. Carol, E. Rizzi, K. WIlliam, A unified theory of elastic degradation and damage 
based on a loading surface, International Journal of Solids and Structures 31 (1994) 2835-2865.

[8] J. Lemaitre, J. Dufailly, Damage measurements, Engineering Fracture Mechanics 28(5/6) (1987) 643-661.

[9] B. Sharp, Eletronic speckle pattern interferometry (espi), Optics and Lasers in Engineering 11 (1989) 241-255.

[10] C. A. Walker, A historical review of moiré interferometry, Experimental Mechanics 34 (1994) 281-299.

[11] T. Chu, W.F., Ranson, M. Sutton, Applications of digital-image-correlation techniques to experimental mechanics, Experimental Mechanics 25 (1985) 232-244.

[12] F. Horkay, D. C. Lin, Mapping the local osmotic modulus of polymer gels, Langmuir 25(15) (2009) 8735-8741.

[13] P. Trtik, J. Kaufmann, U. Volz, On the use of peak-force tapping atmoic force microscopy for qunatification of the local elastic mdoulus in hardend cement paste, Cement and Concrete Research 42 (2012) 215-221.

[14] A. Giannoula, R. S. Cobbold, Mapping the local shear modulus and viscocity using a transient finite-amplitude moduluated radiation force, Ultrasonics 51 (2011) 340351.

[15] M. Sale, P. Rizzo, A. Marzani, Semi-analytical formulation for the guided wavesbased reconstruction of elastic moduli, Mechanical Systems and Signal Processing 25 (2011) 2241-2256.

[16] G. Freihofer, L. Poliah, K. Walker, A. Medina, S. Raghavan, Optical stress probe: in situ stress mapping with Raman and photo-stimulated luminescence spectroscopy, Journal of Instrumentation 5 (2010) P12003. 
[17] I. Hanhan, E. Durnberg, G. Freihofer, P. Akin, S. Raghavan, Portable piezospectropy system: non-contact in-situ stress sensing through high resolution photoluminescent mapping, Journal of Instrumentation 9 (2014) P11005.

[18] G. J. Piermarini, S. Block, J. D. Barnett, R. A. Forman, Calibration of the pressure dependence of the $R_{1}$ ruby fluorescence line to 195 kbar, Journal of Applied Physics 46 (1975) 2774.

[19] R. Christensen, D. Lipkin, D. Clarke, Nondestructive evaluation of the oxidation stresses through thermal barrier coatings using $\mathrm{Cr}^{3+}$ piezospectroscopy, Applied Physics Letters 69 (1996) 3754-3756, .pdf ok.

[20] A. Stevenson, A. Jones, S. Raghavan, Stress-sensing nanomaterial calibrated with photostimulated luminescence emission, Nano Letters 11 (2011) 3274.

[21] G. Freihofer, A. Gupta, A. V. Newkirk, S. Seal, S. Raghavan, Optical stress sensing alumina nanocomposite coatings for aerospace structures, in: AIAA SciTech, 55th AIAA/ASME/ASCE/AHS/SC Structures, Structural Dynamics, and Materials Confrence, 2014.

[22] G. Freihofer, A. Bullock, F. Vaughn, H. Tat, J. Dustin, A. Schlzgen, S. Raghavan, Stress and damage sensing of composite coupons with piezospectroscopic coatings, in: Proceeding of the Society for the Advancement of Material and Process Engineering 2014 conference, Seattle, WA, 2014.

[23] G. Freihofer, J. Dustin, H. Tat, A. Schlzgen, S. Raghavan, Stress and structural damage sensing piezospectroscopic coatings validated with digital image correlation, AIP Advances 5 (2015) 037139.

[24] G. Freihofer, A. Schülzgen, S. Raghavan, Multi-scale mechanics to determine nanocomposite elastic properties with piezospectroscopy, Acta Materialia 81 (2014) 211-218. 
[25] K. Marlett, Hexcel 8552 im7 unidirectional prepreg 190 gsm \& 35\%rc qualification material property data report, Tech. rep., NIAR (2011).

[26] ASTM, D5766/D5766M-11 Standard Test Method for Open-Hole Tensile Strength of Polymer Matrix Composite Laminates, ASTM International, West Conshohocken, PA, 2011.

[27] G. Freihofer, Nanocomposite coating mechanics via piezospectroscopy, Ph.D. thesis, University of Central Florida (2014).

[28] J. He, D. R. Clarke, Determination of the piezospectroscopic coefficients for chromium doped sapphire, Journal of American Ceramic Society 78 (5) (1995) $1347-1353$.

[29] D. Lipkin, D. Clarke, Measurement of the stress in oxide scales formed by oxidation of alumina-forming alloys, Oxidation of Metals 45 (1996) 267.

[30] Q. Ma, D. R. Clarke, Piezospectroscopic determination of residual stresses in polycrystalline alumina, Journal of American Ceramic Society 77 (1994) 298-302.

[31] J. He, I. J. Beyerlein, D. R. Clarke, Load transfer from broken fibers in continous fiber $\mathrm{Al}_{2} \mathrm{O}_{3}-\mathrm{Al}$ composites and dependence on local volume fraction, Journal of the Mechanics and Physics of Solids 47 (1999) 465-502.

[32] P. Marur, R. Batra, G. Garcia, A. Loos, Static and dynamic fracture toughness of epoxy/alumina composite with submicron inclusions, Journal of Materials Science 39 (4) (2004) 1437-1440.

[33] J. Cho, M. Joshi, C. Sun, Effect of inclusion size on mechanical properties of polymeric composites with micro and nano particles, Composites Science and Technology 66 (2006) 1941-1952.

[34] F. Deng, K. Vliet, Prediction of elastic properties for polymer-particle nanocomposites exhibiting an interphase, Nanotechnology 22 (2011) 165703. 
[35] R. G. Munro, Evaluated material properties for a sintered $\alpha$ alumina, J. Am. Ceram. Soc. 80 (1997) 1919-1928.

[36] N. Hansen, H. Schreyer, A thermodynamically consistent framework for theories of elastoplasticity coupled with damage, International Journal of Solids and Structures 31 (1994) 359-389.

[37] J. Reddy, A. Miravete, Practical Analysis of composite laminates, CRC Press, Inc., 1995.

[38] P. Withers, The determination of the elastic field of an ellipsoidal inclusion in a transversely isotropic medium, and its relevence to composite materials, Philosophical magazine A 59 (1989) 759-781.

[39] Y. Zhu, E. Liu, Approximate eshelby tensor for transversely isotropic media, in: Society of Exploration Geophysicists, 2011.

[40] E. Kontou, Micromechanics model for particulate composites, Mechanics of materials 39 (7) (2007) 702-709.

[41] J. Lubliner, Plasticity Theory, Dover Publications, 2008.

[42] A. Stevenson, A. Jones, S. Raghavan, Characterization of particle dispersion and volume fraction in alumina-filled nanocomposites using photo-stimulated luminescence spectroscopy, Polymer 43 (2011) 923-929.

[43] M. Alves, J. Yu, N. Jones, On the elastic modulus degradation in continuum damage mechanics, Computers \& Structures 76 (6) (2000) 703-712.

[44] A. C. Luo, Y. Mou, R. P. Han, A large anisotropic damage theory based on an incrmental complementary energy equivalence model, International Journal of Fracture 70 (1995) 19-34. 
[45] P. P. Camanho, P. Maimí, C. Dávila, Prediction of size effects in notched laminates using continuum damage mechanics, Composites Science and Technology 67 (13) (2007) 2715-2727.

[46] V. Vinogradov, Z. Hashin, Probabilistic energy based model for prediction of transverse cracking in cross-ply laminates, International Journal of Solids and Structures $42(2005) 365-392$.

[47] D. Mollenhauer, E. Iarve, R. Kim, B. Langley, Examination of ply cracking in composite laminates with open holes: A moiré interferometric and numerical study, Composites Part A: Applied Science and Manufacturing 37 (2006) 282-294. 
PS system $\Delta \mathrm{v}$
$\left(\mathrm{cm}^{-1}\right)$

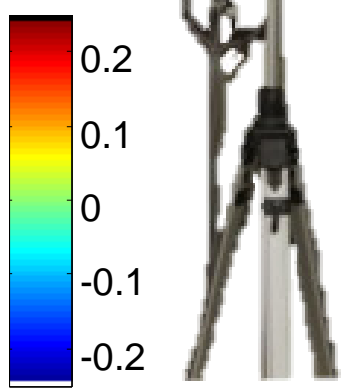

PS shift map

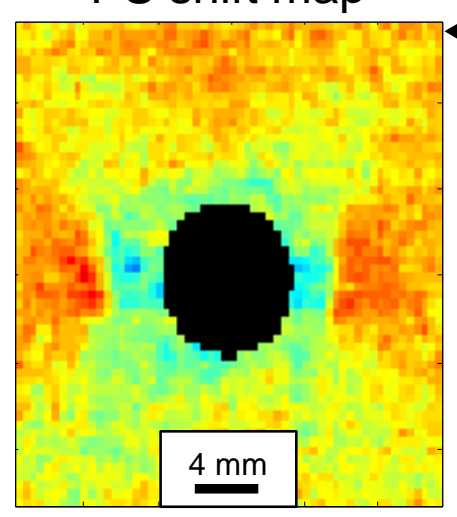

.
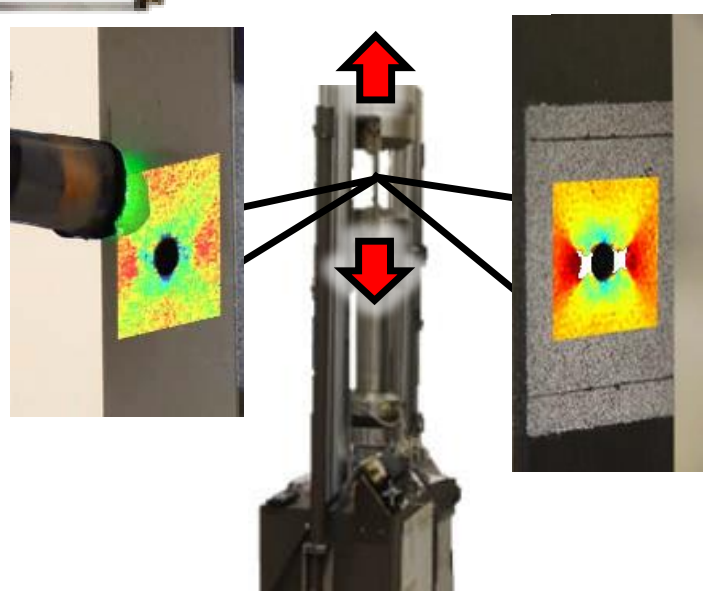

Load History

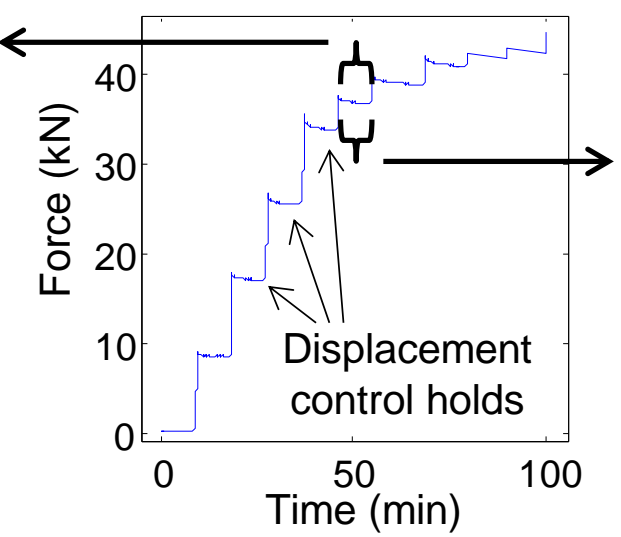

DIC system

$\varepsilon_{1}^{s}+\varepsilon_{2} s$

$(\mathrm{mm} / \mathrm{m})$
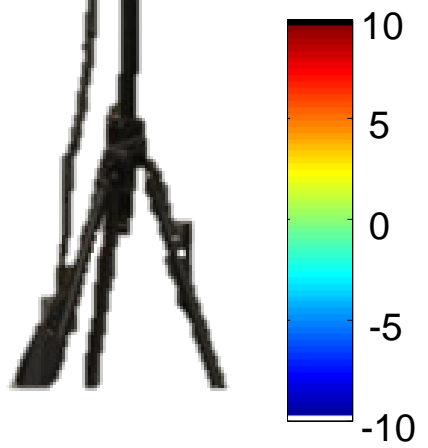

Biaxial strain map

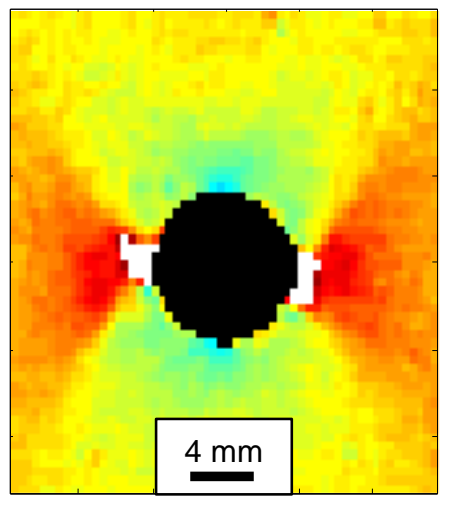


Applied Force

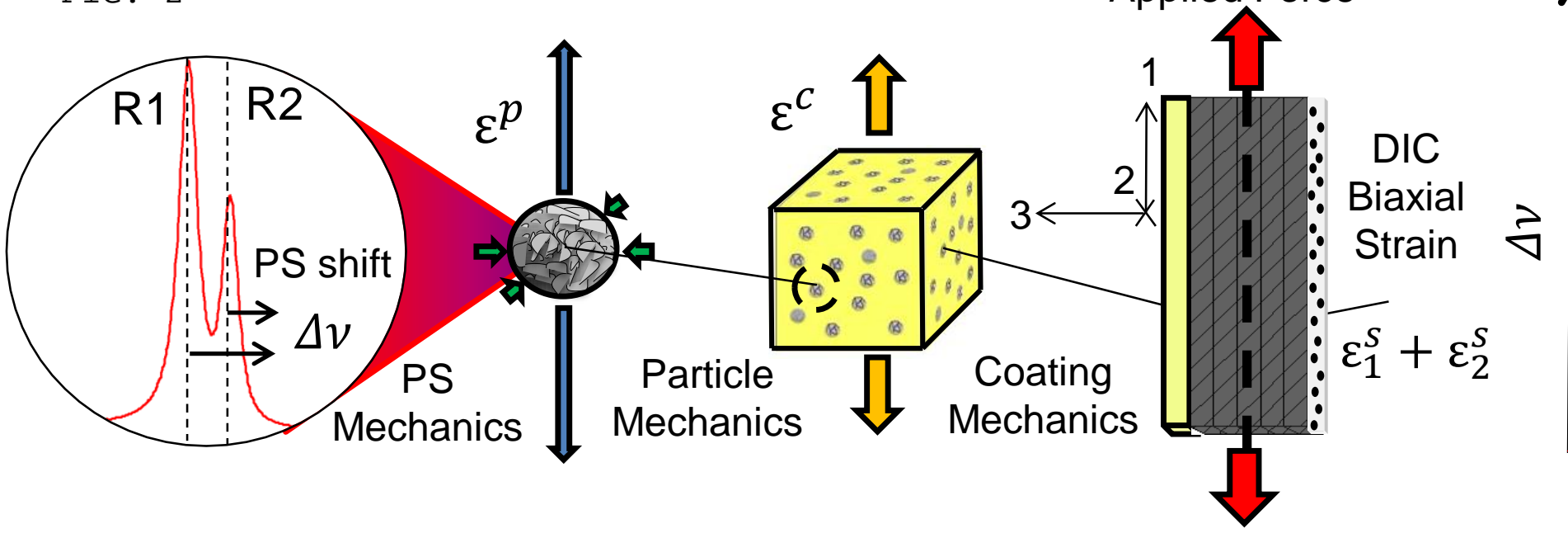

PS response

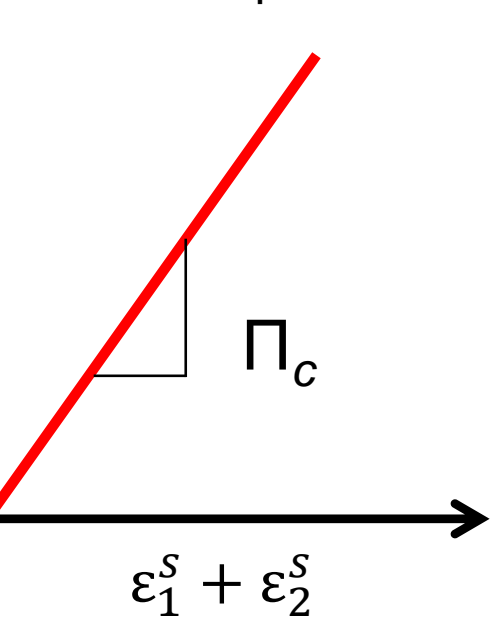




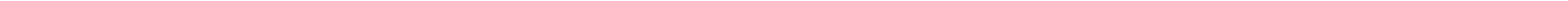



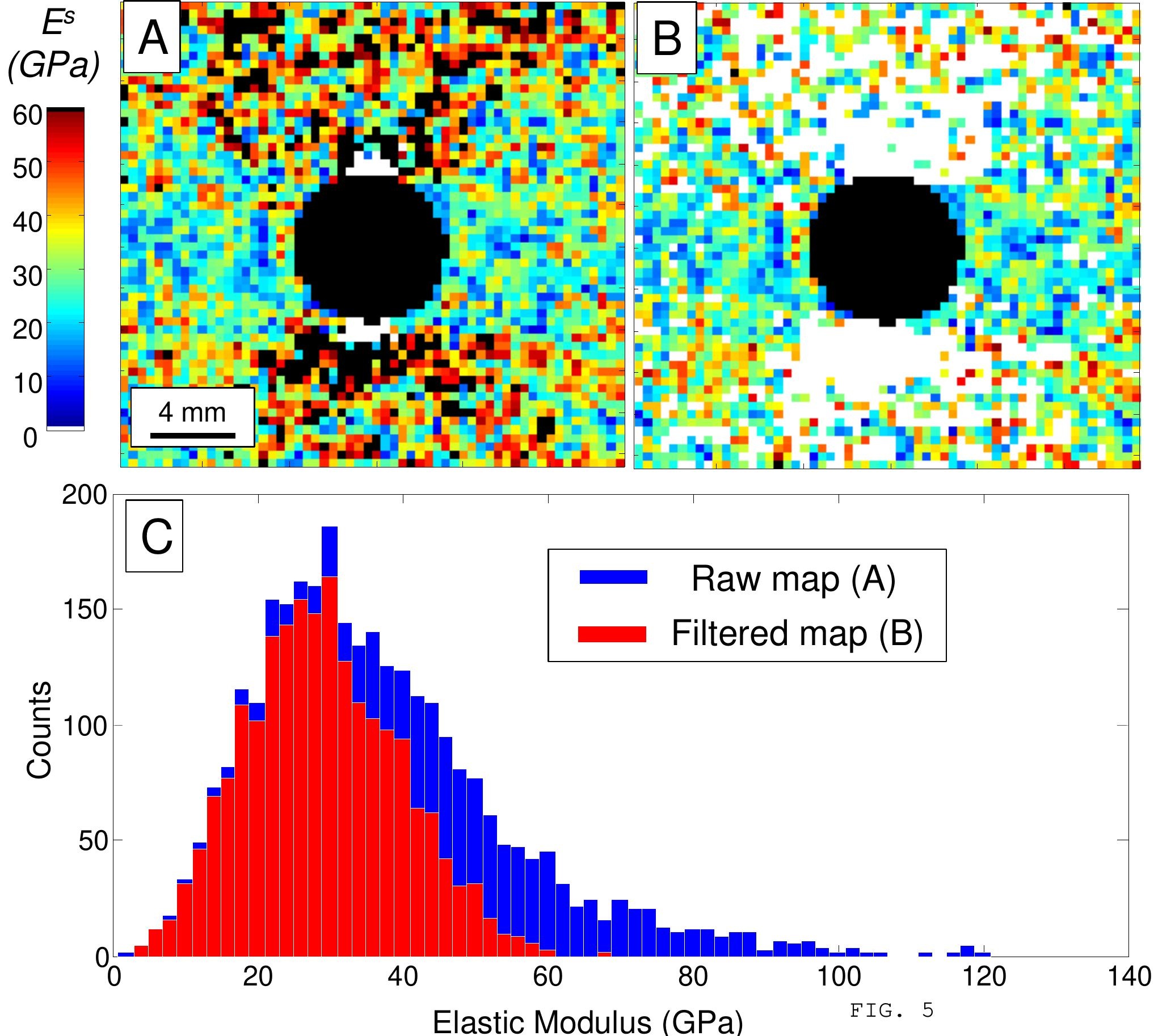


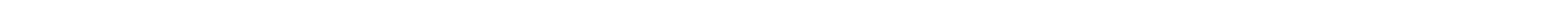


PS system

$\Delta \mathrm{v}$

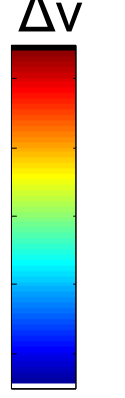

PS shift map
Load frame
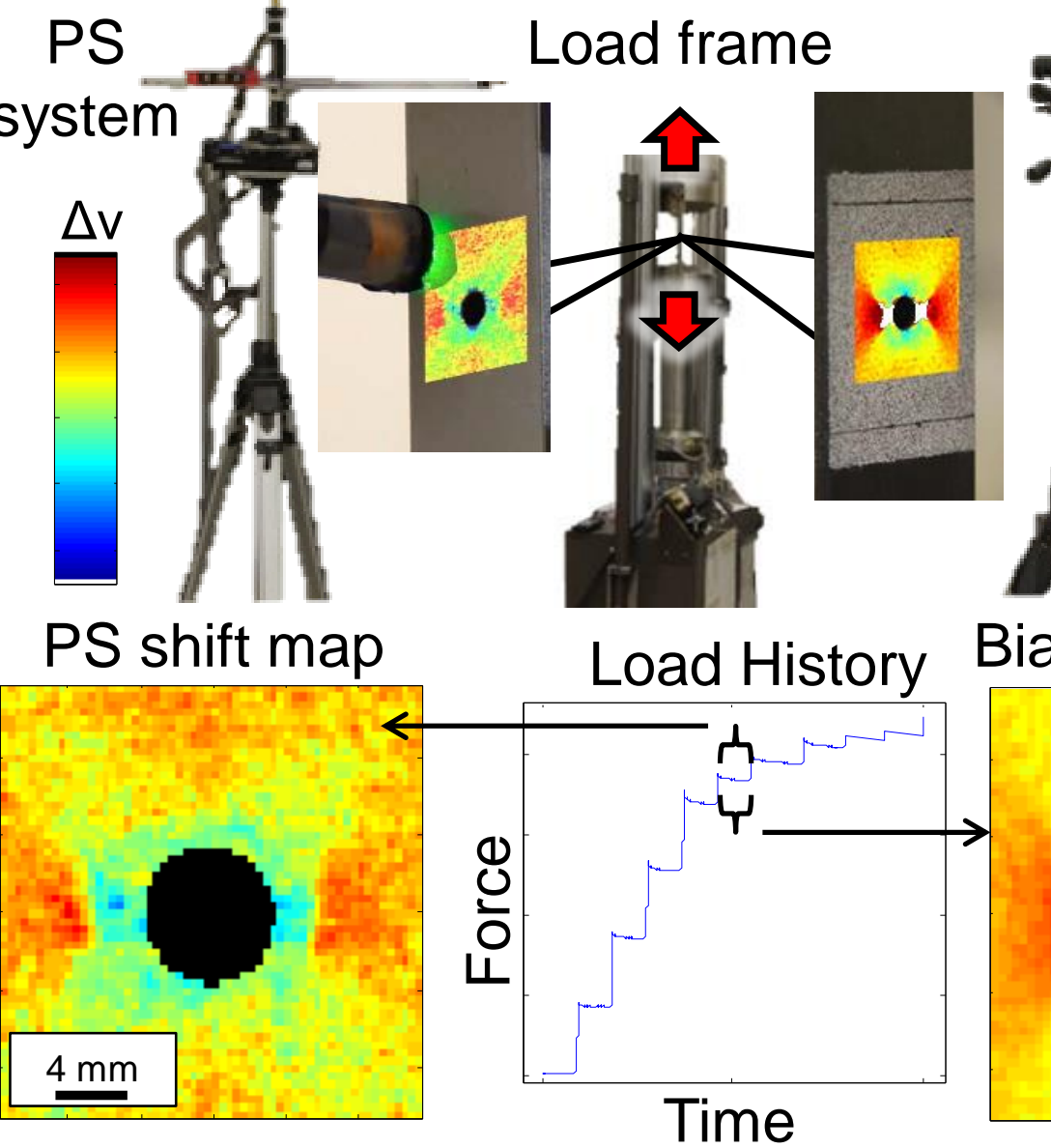

Biaxial strain map system

damage model

$\varepsilon_{1}^{s}+\varepsilon_{2}^{s}$
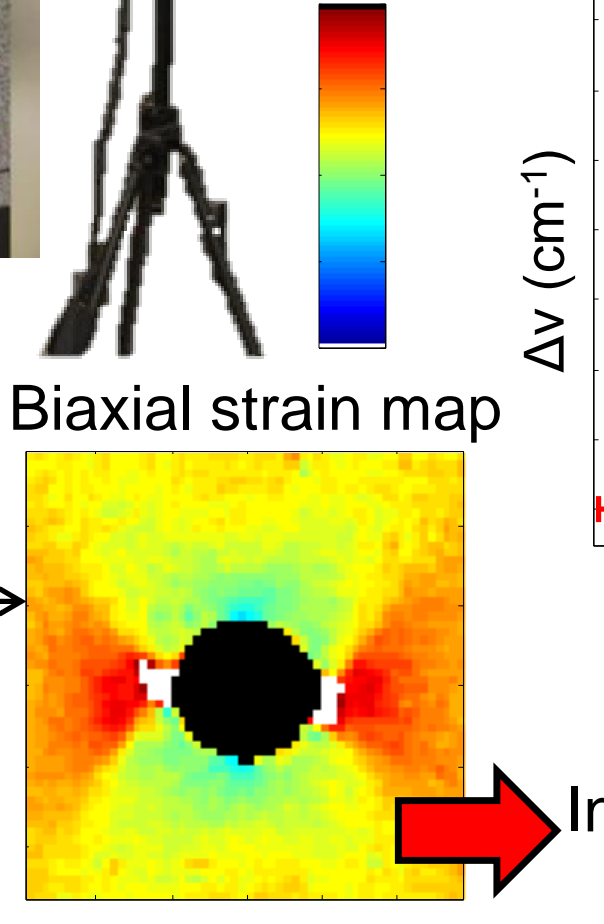

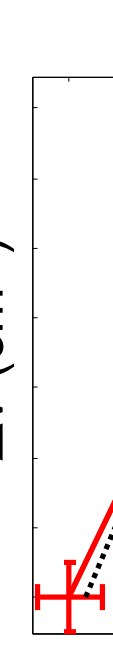

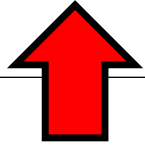

(A)

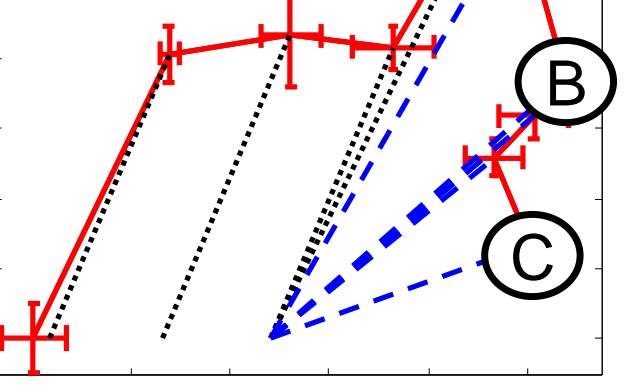

$\varepsilon_{1}{ }^{s}+\varepsilon_{2}{ }^{s}(m m / m)$

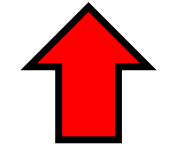

Integrated experimental techniques
Degrading

elastic modulus maps

$1-\tilde{E} / E$

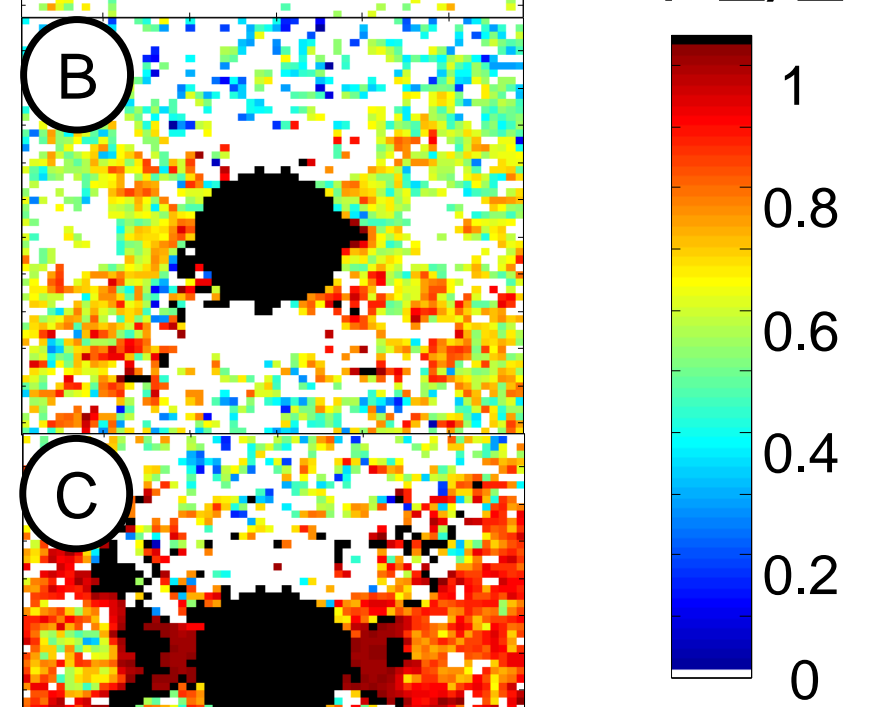

GRAPHICAL ABSTRACT 